\title{
Numerical simulation of the heat-relief capacity of an axisymmetric body in a gas flow
}

\author{
Nikolay Chernov $^{1}$, Alexander Palii ${ }^{2}$, Larisa Tolmacheva $^{2}$, Maya Poklonskaya ${ }^{2 *}$, and Milina \\ Nickolskaya $^{2}$ \\ ${ }^{1}$ Southern Federal University, Nekrasovskiy, 44, Taganrog, 347900, Russia \\ ${ }^{2}$ Politechnical Institute (branch) of DSTU in Taganrog, Petrovskaya str., 109a, Taganrog, 347900, \\ Russia
}

\begin{abstract}
The paper describes a computational experiment and presents the results of numerical modeling of an axisymmetric body with an optimized shape with a minimum aerodynamic drag force as a heat sink in a convective gas flow. The resulting optimized body shape coincides with streamlines, which is the main advantage, since no separation of the flow from the surface is observed in the flow around. Thus, the entire surface area will be the effective surface area of the heat sink, unlike other known body shapes, due to which the temperature of the heat-loaded element placed in the center of the heat sink will decrease.
\end{abstract}

\section{Introduction}

Despite the seeming simplicity of external forms, flow around simple bodies is a very complex process even in isolated conditions. Even more complicated is the flow around and the calculation of the aerodynamic characteristics of bodies, when their simplest geometric shapes are part of even more complex shapes. In this case the mutual influence of individual parts of the body surface on each other is manifested, significantly complicating the initial picture currents. At the same time, the number of geometric parameters that affect the flow structure increases [1].

Aerodynamic characteristics and, in particular, the nature of the flow around bodies of the simplest forms represent a great scientific and practical interest. Bodies of complex shape can always be represented as a set of simpler ones, for which it is easy to investigate and calculate the flow paths, on the basis of which analytical methods for calculating aerodynamic characteristics are compiled. The initial data for this are information obtained from experiments, including a computational experiment with bodies of the simplest shapes [2].

In this paper, numerical modeling is carried out to confirm the theoretical solution to the problem of finding the shape of an optimal aerodynamic body with a minimum aerodynamic drag, which is reduced to calculating the mathematical formula of the curve (streamline) forming a given body by rotation about an axis that coincides with the direction of the flow of a given velocity, which we obtained in our work [3].

\footnotetext{
*Corresponding author: poklonskaya.maya@mail.ru
} 


\section{Problem statement}

In paper [4], we optimized the shape of a body in a gas flow in order to minimize its aerodynamic drag. This shape was obtained by rotating the profile (curve) about the axis coinciding with the direction of the flow of a given speed. The mathematical formula of the curve is described by the following expression [5]:

$$
\left[1-a^{2} /\left(x^{2}+y^{2}\right)\right] y=\text { const }
$$

where $a$ - the radius of the circle.

The radius of the circle a is found according to the magnitude of the dipole moment, determined by the imposition of a uniform flow parallel to the OX axis with a velocity $\mathrm{v}$ on the velocity field of a dipole with a positive moment, which corresponds to the outflow of gas from the dipole towards the incident homogeneous flow (Fig. 1).

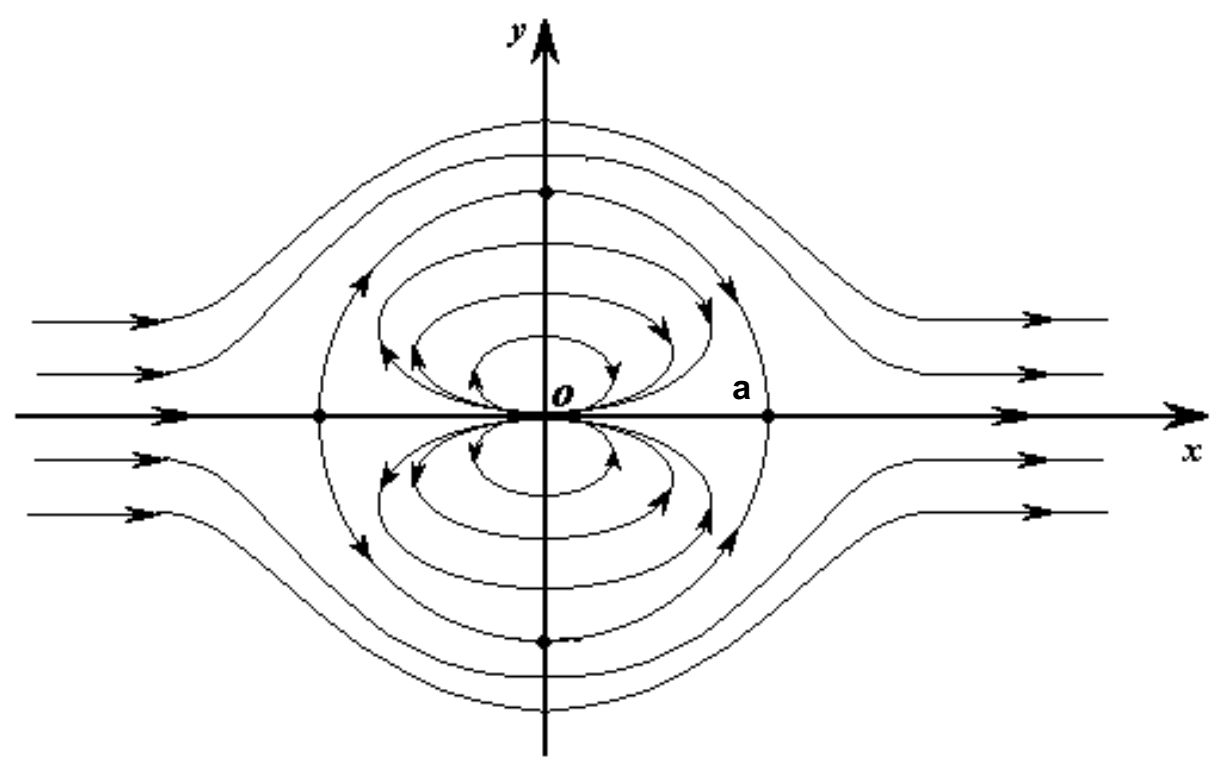

Fig. 1. Combined streamlines when a homogeneous flow is imposed on the velocity field of a dipole with a positive moment.

In this paper, we consider the possibility of using the described body of an optimized shape with a minimum force of aerodynamic resistance as a heat sink in a convective gas flow [6].

When superimposing a homogeneous flow parallel to the OX axis with velocity $\left|v_{\infty}\right|$ and complex potential [7]:

$$
\chi_{1}=\left|v_{\infty}\right| z
$$

to the velocity field of a dipole with a complex potential:

$$
\chi_{2}=m / 2 \pi z
$$


and a positive moment $(m>0)$,which corresponds to the outflow of liquid from the dipole towards the incoming flow, we obtain a complex potential:

$$
\chi=\chi_{1}+\chi_{2}=\left|v_{\infty}\right| z+(m / 2 \pi)(1 / z) .
$$

Separating the imaginary part in this expression, we define the stream function $\Psi$ as:

$$
\Psi=\left|v_{\infty}\right| y-(m / 2 \pi)\left(y /\left(x^{2}+y^{2}\right)\right) .
$$

For the zero current line $\Psi=0$, we get the equation [8]:

$$
\left[\left|v_{\infty}\right|-(m / 2 \pi)\left(1 /\left(x^{2}+y^{2}\right)\right)\right] y=0 .
$$

Choosing the magnitude of the dipole moment equal to:

$$
m=2 \pi a^{2}\left|v_{\infty}\right|,
$$

we get the zero streamline in the form of a circle of radius $a$ centered at the origin and axis $O X$.

The rest of the streamlines are obtained by specifying different values of the constant in the streamline equation (obtained by substituting the value $m=2 \pi a^{2}\left|v_{\infty}\right|$, shown on Fig. 2 [9].

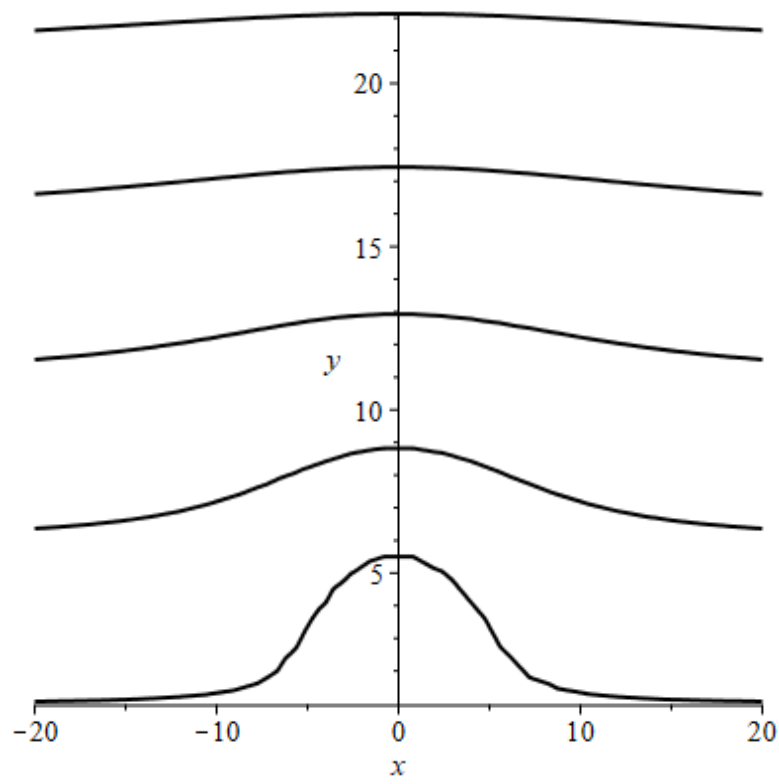

Fig. 2. Change in the shape of streamlines with increasing distance from the bod.

\section{Description of the computational experiment}

In order to now pass from the aerodynamic problem to the thermal one, it is necessary to obtain equi-temperature surfaces corresponding to the optimized form of heat removal in 
the convective flow of a given velocity and a given velocity of heat energy propagation from a point heat source. To do this, we replace the spherical region of zero potential of the dipole field, corresponding to a similar spherical region from a point heat source of double power. In this case, the general character of the aligned streamlines will not change, but aligned equi-temperature lines (surfaces) from the heat source in the convective flow will be obtained [10].

The main advantage of the obtained optimized body shape is that it coincides with the streamlines, thus, when it flows around, the flow separation from the surface will not be observed. Thus, the entire surface area will be the effective surface area of the heat sink, due to which the temperature of the heat-loaded element placed in the center of the heat sink will decrease [11].

To prove this statement, we present the results of numerical modeling carried out in the Ansys Fluent software product to study the temperature of a point heat-loaded element in a heat sink of an optimized shape, calculated for a given convective flow rate and, for example, a spherical one, which is notable for the minimum surface area for equal body volumes. Comparison conditions in a computational experiment are constant for both bodies: volume and shape of the working area; distances from sources, drains and centers of bodies; gas flow rate; masses of bodies, thermal power of the source and other parameters besides the shape of the surface (Fig. 3) [12].

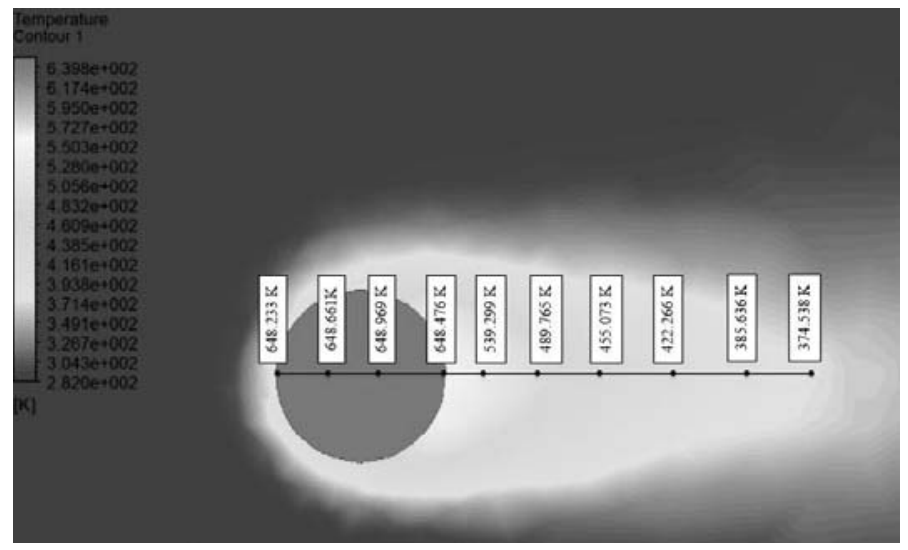

a)

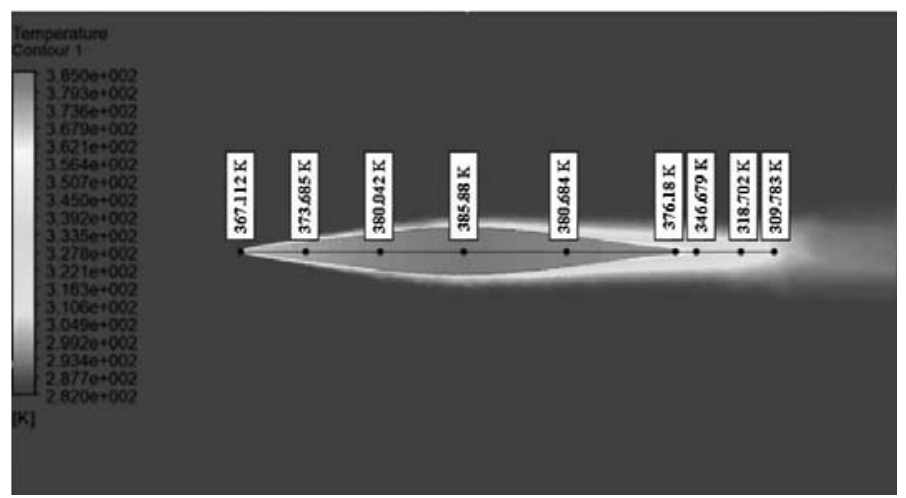

b)

Fig. 3. Distribution of the temperature field in the spherical (a) and optimized axisymmetric (b) heat sinks. 
From Fig. 3 it can be seen that the temperature of the point source in the center of the optimized heat sink (for a given speed - spindle-shaped) is lower than in the spherical one. Given the distribution of the temperature field, one can make an erroneous conclusion that the temperature decrease occurs due to a larger surface area. It should be noted here that it is not the total surface area of the heat sink that is important, but the effective one, that is, taking part in the heat removal by the flow without separating it. Fig. 3 it can be seen that the separation of the flow from the ball occurs approximately at the border of the left and right hemispheres, that is, the right part of the heat sink is practically not flown around. The separation from the optimized heat removal does not occur, therefore, the entire surface area is involved, since the heat removal is made along equi-temperature lines (equipotential surfaces) $[13,14]$.

Changing the experimental conditions by changing the flow rate at which the previous spindle shape will no longer be optimal, we will obtain a significant increase in the temperature of the source as compared to the source in the spherical heat sink [15].

\section{Conclusion}

After changing the conditions of the numerical simulation, the temperature of the source in the spherical heat sink changed insignificantly, and the temperature of the source in the spindle-shaped one increased significantly. This happens due to the fact that the zone of separation of the flow from the spherical heat sink practically does not change, and from the spindle-shaped one is significantly displaced closer to the source. Thus, despite the preservation of the total area of the heat sink, the effective area decreases significantly, and, consequently, the temperature of the heat-loaded element increases.

Ultimately, the efficiency of heat removal can depend on many factors, such as nonlinearity of the flow, the ratio of flow rates and the distribution of heat energy from the source, surface roughness, color, etc. The question can be raised about the equal efficiency of the front and rear parts of the heat sink surface. But the main invariable rule for obtaining an effective shape should remain its fulfillment along equi-temperature surfaces from a source in a convective flow of a given velocity.

\section{References}

1. S.P. Malyukov, A.V. Sayenko, S.S. Zinovev, Mathematical Modelling of Magnetron Sputtering Process During Thin Films Formation, in Proceedings of 2020 International Russian Automation Conference, RusAutoCon (2020)

2. E.L. Houghton, P.W. Carpenter, Steven H. Collicott, D.T. Valentine, Aerodynamics for Engineering Students (Elsevier Ltd, 2013)

3. N.N. Chernov, A.V. Palii, A.M. Maevskiy, V.V. Ignatyev, O.B. Spiridonov, In Proceedings of SPIE - The International Society for Optical Engineering, 11163, 111630K (2019)

4. N.N. Chernov, A.V. Palii, A.V. Sayenko, A.M. Maevskii, Technical Physics Letters, 44(4), 328-330 (2018)

5. A. Townsend, A graduate introduction to numerical methods: From the Viewpoint of Backward Error Analysis (Springer, New York, Heidelberg, 2013)

6. M. Belkin, H. Narayanan, P. Niyogi, Random Structures and Algorithms, 43(4), 407428 (2013)

7. A.V. Palii, A.V. Saenko, V.V. Bespoludin, Engineering Bullet of Don, 2(41) (2016), http://www.ivdon.ru/ru/magazine/archive/n2y2016/3661. 
8. M. Moallemi, J. Clifford, J. Neighbors, et al., Pesce J., Towhidnejad M., In Proceedings of AIAA/IEEE Digital Avionics Systems Conference, 7778002 (2016)

9. N.N. Chernov, A.V. Kovalev, A.V. Palii, et al., IOP Conf. Ser.: Mater. Sci. Eng., 862(3), 032028 (2020)

10. N.N. Chernov, A.V. Palii, V.V. Ignatyev, et al., Advances in Intelligent Systems and Computing, 1224 AISC, 380-387 (2020)

11. A.V. Saenko, S.P. Malyukov, A.V. Paliyi, et al., Engineering Bulletin of Don, 3(46) (2017), http://www.ivdon.ru/ru/magazine/archive/N3y2017/4306.

12. M. Smeaton, R. Vennell, A. Harang, Renewable Energy, 99, 45-56 (2016)

13. W.M. Shibani, M.F. Zulkafli, B. Basuno, IOP Conf. Ser.: Mater. Sci. Eng., 160(1), 012041 (2016)

14. Z. Qi, Y. Wang, Y. Sha, L. Wang, Journal of Beijing University of Aeronautics and Astronautics, 42(12), 2691-2697 (2016)

15. B. Turan, H.F. Oztop, Thermal Science, 16(1), 139-149 (2012) 